\title{
CARCINOMA PAPILAR DE TIROIDES EN QUISTE DEL CONDUCTO TIROGLOSO*
}

\author{
Drs. Patricio Cabané T. ${ }^{1}$, Patricio Gac E. ${ }^{1}$, Francisco Rodríguez M. ${ }^{1}$, \\ Al. Carolina Morales O. ${ }^{2}$, Dr. Juan Aldana L. ${ }^{1}$, Al. Ignacio Boza T. ${ }^{2}$, \\ E.U. Cristina Fernández E. ${ }^{1}$, Dr. José Amat V. ${ }^{1}$ \\ 1 Departamento Cirugía. Hospital Clínico Universidad de Chile. \\ 2 Alumno Facultad de Medicina. Universidad de Chile. Santiago. \\ Chile.
}

\section{Abstract}

\section{Thyroglossal duct cyst with papillary carcinoma}

Introduction: In about 1-2\% of cases of thyroglossal cyst may be neoplastic changes, mostly correspond to papillary thyroid carcinoma $(75-85 \%)$. The aim of this paper is to present 9 cases of papillary thyroid carcinoma in thyroglossal duct cyst. Materials and Methods: Data were recorded retrospectively from patients diagnosed with papillary thyroid carcinoma in thyroglossal duct cyst treated at Hospital Clínico Universidad de Chile between 1999-2014. Results: From 142 cases operated for thyroglossal duct cyst, 9 cases of papillary cancer $(6.34 \%)$ were recorded. The average age was 32 years. The average diameter of the lesion was $4.4 \mathrm{~cm}$ (SD $2.2 \mathrm{~cm}) .8$ patients underwent total thyroidectomy; a simultaneous thyroid cancer was diagnosed in 3 of them. In 6 cases was added iodine therapy. In only one patient a lymph nodal dissection was performed. We do not observe any surgical complication. A solid component in preoperative ultrasonographic study is suspicious of malignancy. The average follow-up time was 85 months. There is no recurrence or mortality in this group of patients. Conclusions: Although the safest long-term management is Sistrunk surgery associated with thyroidectomy and radioiodine in selected cases, these patients must be evaluated by a multidisciplinary group and thyroidectomy should be considered in high surgical volume center, in order to minimize complications.

Key words: Thyroglossal duct, cyst, papillary carcinoma, thyroidectomy.

\section{Resumen}

Introducción: En alrededor de 1-2\% de los casos de quiste tirogloso pueden existir cambios neoplásicos, en su mayoría corresponden a carcinoma papilar de tiroides (75-85\%). El objetivo de este trabajo es presentar 9 casos de carcinoma papilar de tiroides en quiste del conducto tirogloso, en cuanto a su forma de presentación y manejo. Material y Método: Se registraron en forma retrospectiva datos de pacientes con

*Recibido el 4 de agosto de 2014 y aceptado para publicación el 2 de septiembre de 2014.

Los autores no refieren conflictos de interés.

Correspondencia: Dr. Patricio Cabané T. pcabane@hcuch.cl 
diagnóstico de carcinoma papilar de tiroides en quiste del conducto tirogloso atendidos en el Hospital Clínico de la Universidad de Chile entre 1999-2014. Resultados: De 142 casos de pacientes operados por quiste del conducto tirogloso, se registraron 9 casos de cáncer papilar (6,34\%). El promedio de edad de los pacientes fue de 32 años. El diámetro promedio de la lesión fue de 4,4 cm (DS 2,2 cm). Del total, 8 pacientes fueron sometidos a tiroidectomía total, se diagnosticó cáncer de tiroides en 3 de ellos, en 6 se asoció tratamiento con radioyodo. En sólo 1 paciente se realizó una disección linfonodal. El tiempo promedio de seguimiento fue de 85 meses; a la fecha la serie no ha presentado recurrencia ni mortalidad. Conclusiones: Si bien el manejo más seguro a largo plazo es la cirugía de Sistrunk, asociado a una tiroidectomía y eventual radioyodo, la resolución quirúrgica con tiroidectomía asociada debe ser considerada cuando la morbilidad no sea mayor que el beneficio teórico.

Palabras clave: Conducto tirogloso, quiste, carcinoma papilar, tiroidectomía.

\section{Introducción}

El quiste del conducto tirogloso es una de las anomalías más comunes en el desarrollo de la glándula tiroides, también es una de las masas en la línea media del cuello más frecuentes durante la niñez $(75 \%)^{1,2}$. El quiste se desarrolla por una atrofia incompleta del conducto que usualmente ocurre durante la séptima semana de gestación ${ }^{3}$. Remanentes del conducto tirogloso están presentes en el 7\% de la población general ${ }^{1,4}$, en algunos casos $(1-2 \%)^{5-7}$ pueden existir cambios neoplásicos, que corresponden a carcinoma papilar de tiroides en un 75-85\%, el 7\% corresponde a carcinoma mixto papilar-folicular y un $5 \%$ a carcinoma de células escamosas ${ }^{6}$.

Clásicamente el quiste se presenta como asintomático y de crecimiento lento ${ }^{1,3}$. La extirpación del quiste se realiza a través del procedimiento de Sistrunk, técnica que ofrece menor recidiva (3-10\%) que la simple escisión $(30-60 \%)^{8}$. Es en la etapa postoperatoria, en el análisis anatomopatológico del quiste, donde habitualmente se diagnostica el carcinoma papilar de tiroides en quiste tirogloso.

\section{Material y Método}

Se registraron en forma retrospectiva los datos de pacientes con diagnóstico de carcinoma papilar de tiroides en el quiste del conducto tirogloso atendidos en el Hospital Clínico de la Universidad de Chile entre 1999 y 2014. Los casos fueron pesquisados en forma prospectiva desde 2005 a la fecha y en retrospectiva con anterioridad a 2005. Fueron incluidos todos los pacientes con el diagnóstico de carcinoma papilar en quiste del conducto tirogloso, tratados en el Hospital Clínico de la Universidad de Chile en las fechas mencionadas, sin exclusión.

Se registraron datos generales de los pacientes, síntomas y signos de presentación, tipo de estudio y características de imágenes preoperatorias, tipo de cirugía, estudio histopatológico, y evolución.
Los pacientes accedieron a participar en el estudio a través de la firma de un consentimiento informado, durante el estudio se mantuvo la confidencialidad de todos sus antecedentes.

\section{Resultados}

En la Tabla 1 se presentan las características clínicas de cada paciente tratado por cáncer papilar de tiroides en quiste del conducto tirogloso. La edad de presentación promedio fue de 32 años (rango 17-52). La distribución por sexo fue de 5 mujeres y 4 hombres. Entre los años 1999 y 2014 se realizaron 142 cirugías en casos de pacientes con quiste tirogloso en nuestro hospital, de ellos 9 presentaban desarrollo de cáncer tiroideo en el quiste (6,34\%); todos los casos fueron del tipo papilar. Con respecto al estudio imagenológico preoperatorio, en 4 pacientes se realizaron ecografías preoperatorias, 1 paciente $(3 / \mathrm{M})$ se realizó una tomografía computada de cuello y 1 paciente $(9 / F)$ cuenta con una ecografía luego de un año de la cirugía de resección del quiste, en los pacientes restantes no logramos rescatar el estudio imagenológico. De los 4 pacientes con ecografía preoperatoria disponible encontramos 3 con hallazgos sugerentes de un proceso neoplásico, destacando la presencia de un componente sólido intraquístico y microcalcificaciones. En el paciente 3/M se realizó una TC de cuello por el antecedente de una tiroidectomía previa realizada 3 años antes de la presentación del quiste por un cáncer folicular, donde se evidenció un componente sólido sospechoso en el quiste y adenopatías regionales. Para la resección del quiste se utilizó el procedimiento de Sistrunk en 8 de 9 pacientes y resección de Trotter en 1 paciente. Se realizó tiroidectomía total en 8 casos, el paciente $3 / \mathrm{M}$ ya contaba con tiroidectomía y radioyodoterapia a raíz de sus antecedentes. De las 8 tiroidectomías, 4 fueron diferidas, luego de reportarse el hallazgo de cáncer papilar en el quiste posterior a la resección de éste, encontrándose un cáncer sincrónico de tiroides en 2 pacientes. La 
Tabla 1. Características clínicas

\begin{tabular}{|c|c|c|c|c|c|c|}
\hline $\begin{array}{l}\text { Caso/ } \\
\text { Género }\end{array}$ & $\begin{array}{l}\text { Edad } \\
\text { (años) }\end{array}$ & Hallazgos ecográficos & $\begin{array}{c}\text { Técnica } \\
\text { quirúrgica }\end{array}$ & $\begin{array}{c}\text { Estudio } \\
\text { histopatológico }\end{array}$ & $\begin{array}{c}I^{131} \text { post } \\
\text { tiroidectomía }\end{array}$ & $\begin{array}{c}\text { Diámetro } \\
\text { mayor quiste } \\
\text { tirogloso }(\mathrm{cm})\end{array}$ \\
\hline $1 / \mathrm{F}$ & 20 & $\begin{array}{l}\text { Componentes sólidos } \\
\text { intraquísticos múltiples. } \\
\text { Tiroides sin hallazgos }\end{array}$ & $\begin{array}{l}\text { Sistrunk + Ttx } \\
\quad \text { (diferida) }\end{array}$ & $\begin{array}{c}\text { CPT en QCT. } \\
\text { Tiroides (-) }\end{array}$ & $(+)$ & 9 \\
\hline $2 / \mathrm{F}$ & 30 & $\begin{array}{l}\text { Lesión sólida intraquística } \\
\text { vascularizada, con micro- } \\
\text { calcificaciones. Líquido } \\
\text { espeso. Nódulos tiroideos } \\
\text { sospechosos (2) }\end{array}$ & Sistrunk + Ttx & $\begin{array}{c}\text { CPT en QCT. } \\
\text { Tiroides (-) }\end{array}$ & $(-)$ & 2 \\
\hline $3 / \mathrm{M}$ & 40 & $\begin{array}{l}\text { TC: LN sospechosos. } \\
\text { Lesión sólida vasculari- } \\
\text { zada }\end{array}$ & $\begin{array}{l}\text { Sistrunk + Resec- } \\
\text { ción Ganglionar } \\
\text { grupos I, II y III D }\end{array}$ & $\begin{array}{l}\text { CPT en QCT. } \\
\text { LN (-). Cá folicular } \\
\text { tiroideo previo }\end{array}$ & $(+)$ & 4,5 \\
\hline $4 / \mathrm{M}$ & 45 & Quiste tirogloso simple & $\begin{array}{l}\text { Sistrunk }+ \text { Ttx } \\
\quad \text { (diferida) }\end{array}$ & $\begin{array}{c}\mathrm{CPT} \text { en } \mathrm{QCT}+ \\
\text { Cá papilar tiroideo }\end{array}$ & $(+)$ & 5 \\
\hline $5 / \mathrm{M}$ & 35 & $\begin{array}{l}\text { Componente sólido } \\
\text { intraquístico con } \\
\text { calcificaciones. Tiroides } \\
\text { sin nódulos ni adenopatías }\end{array}$ & Sistrunk + Ttx & $\begin{array}{c}\text { CPT en QCT. } \\
\text { Tiroides (-) }\end{array}$ & $(+)$ & 6 \\
\hline $6 / \mathrm{F}$ & 17 & Sin imagenología & $\begin{array}{l}\text { Sistrunk + Ttx } \\
\quad \text { (diferida) }\end{array}$ & $\begin{array}{c}\text { CPT en QCT. } \\
\text { Tiroides (-) }\end{array}$ & $(+)$ & 5 \\
\hline 7/M & 31 & Sin imagenología & $\begin{array}{l}\text { Resección de } \\
\text { Trotter }\end{array}$ & CPT en QCT & $(-)$ & 2,5 \\
\hline $8 / \mathrm{F}$ & 52 & Sin imagenología & Sistrunk + Ttx & $\begin{array}{c}\text { CPT en QCT + } \\
\text { Cá micropapilar } \\
\text { tiroideo + Tiroiditis } \\
\text { Crónica de } \\
\text { Hashimoto }+ \\
\text { Adenoma folicular }\end{array}$ & $(+)$ & 2 \\
\hline $9 / \mathrm{F}$ & 21 & $\begin{array}{l}\text { Un años post cirugía: } \\
\text { nódulos tiroideos } \\
\text { hipoecogénicos de } 6 \mathrm{~mm} \\
\text { con vascularización }\end{array}$ & $\begin{array}{l}\text { Sistrunk }+ \text { Ttx } \\
\quad \text { (diferida) }\end{array}$ & $\begin{array}{c}\text { CPT en QCT. } \\
\text { Tiroides (-) }\end{array}$ & $(-)$ & 3,8 \\
\hline
\end{tabular}

Ttx: Tiroidectomía total; CPT en QCT: Cáncer papilar de tiroides en quiste del conducto tirogloso.

Figura 1 muestra la macroscopia de una operación de Sistrunk asociada a una tiroidectomía total. En esta se debe tener especial atención en resecar el lóbulo piramidal y evitar dejar tejido remanente. De los 3 pacientes con cáncer tiroideo en la glándula tiroides, dos fueron del tipo papilar con presentación sincrónica al cáncer papilar del quiste del conducto tirogloso, y uno folicular con presentación previa a este. De los 8 pacientes sometidos a tiroidectomía, 6 pacientes recibieron radioyodoterapia. La resección ganglionar fue realizada sólo en el paciente $3 / \mathrm{M}$, por la presencia de linfonodos sospechosos en la TC preoperatoria y el antecedente de cáncer folicular de tiroides operado, resultando negativos al estudio histopatológico para metástasis de cáncer tiroideo. El tamaño promedio del quiste resecado fue de 4,4 cm (DS: 2,2 cm). El tiempo promedio de seguimiento de los pacientes fue de 85 meses, a la fecha ningún paciente ha presentado recidiva y tampoco se ha reportado mortalidad en esta serie.

\section{Discusión}

A pesar de las modernas técnicas diagnósticas, el carcinoma es poco pesquisado en la etapa preoperatoria, generalmente es asintomático no ofreciendo ninguna pista para su diagnóstico ${ }^{1,3}$. Es muy difícil de identificar al ultrasonido e incluso a través de 


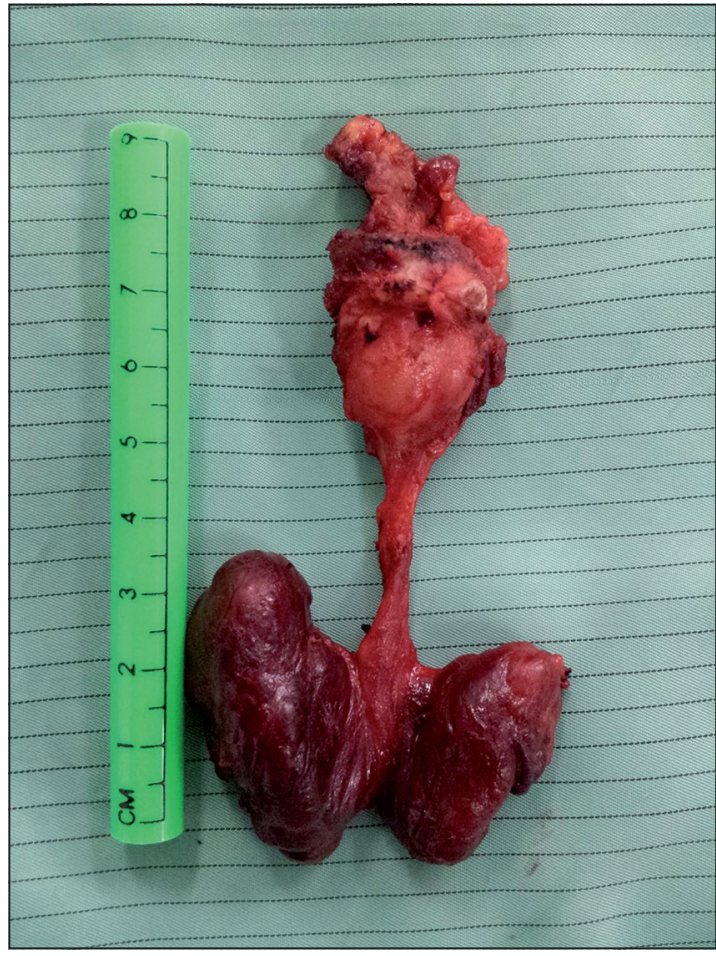

Figura 1. Ejemplo de Macroscopia de pieza operatoria posterior a Operación de Sistrunk más Tiroidectomía Total asociada. Se ve la resección completa del Conducto tirogloso, segmento de hueso hioides, quiste tiroigloso, lóbulo piramidal de tiroides y glándula tiroides.

PAAF; el hallazgo al ultrasonido de una masa intramural, especialmente con microcalcificaciones, es altamente sugerente de un carcinoma ${ }^{10}$. La técnica de PAFF tiene un gran porcentaje de falsos negativos, descrito en un $47 \% \%^{4}$, esto se debe a la hipocelularidad de la muestra producto de la dilución por el contenido quístico. Generalmente lo primero que se extrae es fluido quístico, por ello es importante repetir el procedimiento y aspirar, en caso de existir, una región sólida residual. Se aconseja repetir la PAAF hasta obtener una muestra apropiada, alrededor de 2-3 veces ${ }^{2,6}$. Un diagnóstico preoperatorio permitiría planificar la cirugía del quiste, ya que estos pacientes requieren una exploración acuciosa en búsqueda de cáncer tiroideo y de metástasis en linfonodos?

El origen del carcinoma en quiste tirogloso es aún cuestionado y se debate entre dos opciones, la primera es que sería un cáncer primario del quiste, la segunda plantea que sería una metástasis de un cáncer primario de la glándula tiroides ${ }^{9,10}$. La presencia de folículos tiroideos (hasta en un 62\%) en el conducto del tirogloso ${ }^{11}$ apoyan la hipótesis de novo, ya que tendrían el mismo potencial para la malignización que el tejido de la glándula tiroides. Otra evidencia que apoya la teoría de novo es que no hay presencia de células parafoliculares en el tejido ectópico de tiroides encontrado en el quiste ${ }^{2}$, a la fecha ningún caso de carcinoma medular de tiroides en un quiste tirogloso ha sido reportado ${ }^{10}$. En nuestros casos clínicos existe evidencia que apoya el origen de novo, particularmente en el paciente $3 / \mathrm{M}$, cuya evolución y manejo difiere de otros pacientes. En su caso existe el antecedente de un cáncer folicular de tiroides operado tres años antes de la presentación del quiste, al realizarse la extirpación del quiste tirogloso se determinó que el carcinoma correspondía histológicamente a un cáncer papilar tiroideo. Al relacionar ambos hechos se hace evidente que el carcinoma encontrado en el quiste no pudo haber sido producto de una metástasis de la glándula tiroides, constituyendo una evidencia a favor de la teoría de novo y en contra de la teoría que intenta explicar el origen del carcinoma como una metástasis de la glándula tiroides.

Este debate, junto con los pocos casos reportados en la literatura hace aún más difícil establecer un consenso en el manejo del cáncer papilar en quiste del conducto tirogloso. Series recientes ${ }^{3,8,11}$ han reportado la co-existencia de carcinoma en el quiste del conducto tirogloso y en la glándula tiroides hasta en un 56-66\% de los pacientes. Este hallazgo plantea la posibilidad de la presencia de una enfermedad multifocal que se desarrolla de manera sincrónica en la glándula tiroides y en el quiste del conducto tirogloso. Frente a este panorama se plantea la necesidad de encontrar un acuerdo en el manejo de la patología, y así poder decidir el manejo quirúrgico de la glándula tiroides y de las metástasis en linfonodos en pacientes con carcinoma papilar de tiroides en el quiste tirogloso. Se reportan distintas recomendaciones dependiendo de la serie. Miccoli y cols. ${ }^{9}$, recomiendan la extracción del quiste mediante el procedimiento Sistrunk junto con una tiroidectomía total en todos los pacientes, además de la resección de linfonodos aumentados de tamaño si existiesen, esto garantizaría la remoción completa del tumor y el correcto seguimiento del paciente. Se deben considerar los riesgo de una tiroidectomía total como son la hipocalcemia (1-5\%) y la lesión del nervio laríngeo recurrente $(1-2 \%)^{2}$, además de la pérdida de la función de la glándula que implica una dependencia farmacológica de por vida.

Se han intentado identificar ciertos factores que podrían indicar mayor agresividad del carcinoma papilar en quiste tirogloso, para dar sustento a las recomendaciones con respecto a su manejo. En relación a esto, distintas series ${ }^{12-14}$ recomiendan la tiroidectomía total cuando el tamaño del tumor es mayor a $1 \mathrm{~cm}$ o cuando existe invasión de la pared 
del quiste, ya que se asociaría a una mayor agresividad del carcinoma. Plaza y cols. ${ }^{2}$, proponen un algoritmo de tratamiento y creen que es muy difícil justificar algo más invasivo que el procedimiento de Sistrunk para los pacientes que cumplen los siguientes criterios: 1) Menor de 45 años, sin historia de irradiación en el cuello en su niñez; 2) glándula tiroides clínica o radiológicamente normal; 3 ) tumor menor a $1,5 \mathrm{~cm}$ de diámetro; 4) ausencia de márgenes positivos; 5) ausencia de invasión en la pared del quiste; 6) ausencia de metástasis en linfonodos. En estos pacientes de bajo riesgo se sugiere control anual con TSH y ecografía tiroidea, además de mantener la TSH en rango de supresión (0,5-1 mIU/L). En el resto de los pacientes de alto riesgo se sugiere el procedimiento de Sistrunk con tiroidectomía total, luego se recomienda realizar ablación de los restos tiroideos con yodo radioactivo, mantener TSH en rangos de supresión y controlar anualmente con niveles de tiroglobulina.

A pesar de la incertidumbre con respecto al manejo, el pronóstico del cáncer papilar en quiste tirogloso es excelente ${ }^{14,15}$, similar al cáncer papilar de tiroides alcanza tasas de remisión que superan el $95 \%$, con una incidencia de metástasis a distancia en menos del $2 \%$ de los casos ${ }^{14}$.

La invasión de la pared del quiste, se ha reportado en alrededor de un $45 \%$ de los $\operatorname{casos}^{5,16}$, dado el buen pronóstico del carcinoma y la alta frecuencia de invasión de la pared del quiste es cuestionable el hecho de que efectivamente se asocie a mayor agresividad. El frecuente hallazgo podría deberse al origen del quiste, que probablemente sería en las islas de tejido ectópico tiroideo encontradas adyacentes a la pared del quiste y no en el revestimiento del quiste ${ }^{16}$.

Dada la rareza del cáncer papilar en quiste tirogloso, técnicamente es muy difícil identificar factores de agresividad, por ello se ha tratado de encontrar similitudes con el cáncer papilar de tiroides, que está ampliamente estudiado y cuenta con recomendaciones aceptadas. En su serie clínica Dana Hartly cols. ${ }^{11}$, identificaron diversas similitudes entre ambas patologías, en cuanto a multifocalidad, compromiso de linfonodos, metástasis y pronóstico. Proponen que el cáncer papilar en quiste tirogloso no difiere del cáncer papilar de tiroides, por ello se deberían seguir las guías de recomendación de un cáncer diferenciado de tiroides. Un hallazgo relevante de su serie clínica es que el compromiso de linfonodos no se relacionaría con la probabilidad de encontrar otros focos de cáncer en tiroides. La extensión local a partes blandas ha sido reportada en un $55 \%{ }^{17}$, el significado de este hallazgo en cuanto al pronóstico es aún desconocido y plantea la importante duda sobre la posible realización de tiroidectomía total en estos pacientes ${ }^{18}$. Con respecto al tamaño del quiste, lo estudiado en niños con carcinoma papilar en quiste tirogloso, indicaría que no hay una relación entre tamaño y malignidad, Peretz y cols. ${ }^{16}$, encontraron tamaños similares para quistes benignos y malignos en niños (0-18 años).

La serie clínica y revisión de Patel y cols. ${ }^{5}$, luego de un seguimiento promedio de 71 meses, arrojó que el único factor pronóstico relevante en los pacientes con cáncer papilar en quiste tirogloso sería la extensión de la cirugía del quiste (recomendada mediante la operación de Sistrunk), la adición de tiroidectomía total a la cirugía de Sistrunk o el hallazgo de cáncer diferenciado de tiroides no tuvo impacto en el pronóstico de los pacientes.

El carcinoma papilar de tiroides en el quiste del conducto tirogloso es una patología muy poco frecuente, siendo la gran parte diagnosticada de manera incidental luego de la extirpación del quiste. La evidencia apunta a que este es un cáncer primario y no una metástasis de la glándula tiroides, existe un importante porcentaje de pacientes que podrían presentar el carcinoma en el quiste y en la glándula de manera sincrónica, a pesar de no presentar hallazgos en la ecografía tiroidea. Estos hechos hacen que el debate de cómo manejar quirúrgicamente la patología se mantenga. Las recomendaciones apuntan a estratificar a los pacientes según su riesgo para el manejo adecuado de la patología. En nuestra serie podemos destacar la importancia del hallazgo de un componente sólido en la ecografía para plantear el diagnóstico.

Sin embargo, el seguimiento más seguro es completando el tratamiento con tiroidectomía y eventual radioyodo. Cuando se realice el diagnóstico de carcinoma en quiste tirogloso se debe realizar una exhaustiva revisión de la glándula tiroides y de los linfonodos, ya sea durante la cirugía o posterior a esta, clínica y radiológicamente. Se debe tener en cuenta que la evolución del carcinoma y de sus posibles consecuencias es prologada, por lo que el control y seguimiento de los pacientes es esencial en mantener el buen pronóstico de la patología.

La resolución quirúrgica con tiroidectomía asociada debe ser considerada cuando la morbilidad no sea mayor que el beneficio teórico. Por esto debe ser estudiado caso a caso por un equipo multidisciplinario con amplia experiencia en el manejo del cáncer de tiroides.

\section{Referencias}

1. Tharmabala M, Kanthan R. Incidental thyroid papillary carcinoma in a thyroglossal duct cyst-management dilemmas. International Journal of Surgery Case Reports 2013;4:58-61. 
2. Plaza C, López M, Carrasco C, Meseguer L, Perucho A. Management of Well-Differentiated Thyroglossal Remnant Thyroid Carcinoma: Time To Close the Debate? Report of Five New Cases and Proposal of a Definitive Algorithm for treatment. Ann Surg Oncol. 2006;13:745-52.

3. Pellegriti G, Lumera G, Malandrino P, Latina A, Masucci R, Scollo C, et al. Thyroid Cancer in Thyroglossal Duct Cyst Requires a Specific Approach due to its Unpredictable Extension. J Clin Endocrinol Metal. 2013;98:458-65.

4. Yang Y, Haguir S, Wanamaker J, Powers C. Diagnosis of papillary carcinoma in a thyroglossal duct cyst by fine-needle-aspiration biopsy. Arch Pathol Lab Med. 2000;124:139-42.

5. Patel S, Escrig M, Shaha A, Singh B, Shah J. Management of well-differentiated thyroid carcinoma presenting with thyroglossal duct cyst. J Surg Oncol. 2002;79:134-9.

6. Aculate N, Jones H, Bansal A, Ho M. Papillary carcinoma within a thyroglossal duct cyst: significance of a central solid component on ultrasound imaging. $\mathrm{Br} \mathrm{J}$ Oral Maxillofac Surg. 2014;52:277-8.

7. Hakeem A, Hakeem I, Ahmed R, Kirmani O. Papillary carcinoma in a thyroglossal duct cyst with neck nodal metastases. Thyroid Res Pract. 2013;10:20-2.

8. Chrisoulidou A, Iliadou PK, Doumala E, Mathiopoulou L, Boudina M, Alevizaki, et al. Thyroglossal duct cyst carcinomas: is there need for a thyroidectomy? Hormones 2013;12:522-8.

9. Miccoli P, Minuto M, Galleri D, Puccini M, Berti P. Extent of Surgery in Thyroglossal Duct Carcinoma: Reflections on a series of eighteen cases. Thyroid 2004;14:121-3.

10. Choi Y, Kim T, Song D, Hong S, Jang E, Jeon M, Han
W, et al. Papillary Thyroid carcinoma arising from a thyroglossal duct cyst: a single institution experience. Endocr J. 2013;60:665-70.

11. Hartl D, Al Ghuzlan A, Chami L, Leboulleux S, Schlumberger M, Travagli JP. High rate of multifocality and occult lymph node metastases in papillary thyroid carcinoma arising in thyroglossal duct cyst. Ann Surg Oncol. 2009;16:2595-601.

12. Senthilkumar R, Neville J, Aravind R. Malignant thyroglossal duct cyst with synchronous occult thyroid gland papillary carcinoma. Indian J Endocr Metab. 2013; 17:936-8.

13. Agarwal K, Puri V, Singh S. Critical appraisal of FNAC in the diagnosis of primary papillary carcinoma arising in thyroglossal cyst: A case report with review of literature on FNAC and its diagnostic pitfalls. J Cytol. 2010;27:22-5.

14. Park MH, Yoon JH, Jegal YJ, Lee JS. Papillary Thyroglossal Duct Cyst Carcinoma with Synchronous Occult Papillary Thyroid Microcarcinoma. Yonsei Med J. 2010;51:609-11.

15. Vijay R, Rajan K, Feroze M. Innaparent twin malignancy in thyroglossal cyst: Case Report. World J Surg Oncol. 2003;1:15.

16. Peretz A, Leiberman E, Kapelushnik J, Hershkovitz E. Thyroglossal Duct Cyst carcinoma in children: case presentation and review of the literature. Thyroid 2004; 14:777-85.

17. Manipadam J, Manipadam M, Thomas E, Michael R, Ramakant P, Abraham D, et al. WJOES 2011;3:59-63.

18. Basu S, Shet T, Borges AM. Outcome of primary papillary carcinoma of thyroglossal duct cyst with local infiltration to soft tissues and uninvolved thyroid. Indian J Cancer 2009;46:169-70. 